\title{
Mass-redshift Degeneracy for the Gravitational-wave Sources in the Vicinity of Supermassive Black Holes
}

\author{
Xian Chen, ${ }^{1,2 \star ~ S h u o ~} \mathrm{Li}^{3}$ and Zhoujian $\mathrm{CaO}^{4}$ \\ ${ }^{1}$ Astronomy Department, School of Physics, Peking University, 100871 Beijing, China \\ ${ }^{2}$ Kavli Institute for Astronomy and Astrophysics at Peking University, 100871 Beijing, China \\ ${ }^{3}$ National Astronomical Observatories and Key Laboratory of Computational Astrophysics, Chinese Academy of Sciences, \\ $20 A$ Datun Rd., Chaoyang District, Beijing 100012, China \\ ${ }^{4}$ Institute of Applied Mathematics, Academy of Mathematics and Systems Science, Chinese Academy of Sciences, Beijing 100190, China
}

14 January 2019

\begin{abstract}
Retrieving the mass of a gravitational-wave $(\mathrm{GW})$ source is a fundamental but difficult problem because the mass is degenerate with redshift. In astronomy, three types of redshift exist, namely cosmological, Doppler, and gravitational redshift, but the latter two are normally too weak to affect the observation. In this Letter, we show that the current astrophysical models allow binary black holes (BBHs) to merge within 10 Schwarzschild radii of a supermassive black hole (SMBH). We find that in this case both the Doppler and gravitational redshift are significant, and in the most extreme condition they could increase the "apparent" black-hole mass and distance by a factor of $1.9-3.4$. We show that such a factor is consistent with the distribution in the distance-mass diagram of the ten BBHs detected so far by LIGO/Virgo. We also discuss the difficulties of this redshift scenario caused by the low event rate predicted by the current models, as well the potential solutions.
\end{abstract}

Key words: black hole physics - gravitational waves - methods: analytical - stars: kinematics and dynamics - galaxies: nuclei

\section{INTRODUCTION}

The Laser Interferometer Gravitational-wave Observatory (LIGO) and the Virgo detectors have detected gravitational waves (GWs) from ten merging binary black holes (BBHs) (The LIGO Scientific Collaboration \& The Virgo Collaboration 2018a,b). Interestingly, eight of these binaries contain black holes (BHs) more massive than 20-30 $M_{\odot}$. Such a large mass has not been previously detected in X-ray binaries (McClintock et al. 2014; Corral-Santana et al. 2016) and is $2-3$ times greater than the conventional mass for stellar BHs, $10 M_{\odot}$. Although the detected high mass can be reconciled with the current $\mathrm{BH}$ formation models (Abbott et al. 2016), the absence of $20-30 M_{\odot} \mathrm{BHs}$ in X-ray binaries is more difficult to explain.

One possible solution is that we have significantly overestimated the masses of the GW sources. Its theoretical basis is the well-known degeneracy between mass and redshift: By analysing GW data one derives only the redshifted mass $m(1+z)$, which is greater than the rest mass $m$ by a redshift factor of $1+z$ (Schutz 1986). Since cosmological redshift is omnipresent, it could have caused the aforementioned

^ E-mail: xian.chen@pku.edu.cn discrepancy if what LIGO/Virgo have observed so far were mostly strongly-lensed $\mathrm{BBHs}$ residing at large cosmological distances (Smith et al. 2018; Broadhurst et al. 2018). This scenario, nevertheless, may not explain all the eight detections because it predicts an inverse correlation between the "apparent" masses and distances of the BBHs which is contradictory to what have been observed (The LIGO Scientific Collaboration \& The Virgo Collaboration 2018a).

Besides cosmological redshift, in astronomy there exist also Doppler and gravitational redshifts. The latter two have not been considered as the cause of the observed high masses because in the conventional view of $\mathrm{BBH}$ formation the centre-of-mass (COM) velocity is small relative to the speed of light $(c)$ and the potential energy induced by the environment is negligible (Abbott et al. 2016; Amaro-Seoane $\&$ Chen 2016). However, the conventional view may not be complete in light of the recent progress made by the studies of the stellar dynamics in galactic nuclei.

Recent studies showed that the merger rate of BBHs is enhanced in nuclear star clusters (NSCs) due to the presence of supermassive black holes (SMBHs). The enhancement is caused by the following factors. (1) Stellar-mass BHs are more easily retained in NSCs with SMBHs because the large escape velocity is large (Miller \& Lauburg 2009). (2) A dy- 
namical effect called "mass segregation" increases the density of BHs around SMBHs (Bahcall \& Wolf 1976; Morris 1993; Miralda-Escudé \& Gould 2000; Freitag et al. 2006; Hopman \& Alexander 2006; Alexander \& Hopman 2009; Amaro-Seoane et al. 2004). (3) SMBHs can tidally capture BBHs to bound orbits (Addison et al. 2015; Chen \& Han 2018). (4) Tidal perturbation by the SMBHs can enhance the merger rate of $\mathrm{BBHs}$ through a mechanism called the "Lidov-Kozai effect" (Antonini \& Perets 2012; Prodan et al. 2015; Stephan et al. 2016; VanLandingham et al. 2016; Liu et al. 2017; Petrovich \& Antonini 2017; Bradnick et al. 2017; Hoang et al. 2018; Arca-Sedda \& Gualandris 2018). (5) If the environment is gas-rich, as will be the case in an active galactic nucleus (AGN), BBHs can grow and merge more rapidly (Syer et al. 1991; Bellovary et al. 2016; Bartos et al. 2017; Stone et al. 2017; McKernan et al. 2018).

The results of these earlier studies suggest that it is possible a fraction of, if not all, BBH mergers happen in the vicinity of SMBHs. This possibility motivates us to revisit the problem of mass-redshift degeneracy, taking especially the Doppler and gravitational redshift into account. Thoughout the Letter, we adopt the convention $G=c=1$.

\section{POSSIBLE FORMATION SCENARIOS}

We first investigat the distance $r$ relative to a $\mathrm{SMBH}$ where $\mathrm{BBH}$ merger could possibly happen. We find in the literature two mechanisms that could deliver BBHs to $r \lesssim 10 R_{S}$, where $R_{S}$ is the Schwarzschild radius of the SMBH.

The first mechanism is tidal capture (Addison et al. 2015; Chen \& Han 2018). The necessary condition is that a $\mathrm{BBH}$, initially far from and gravitationally unbound to a SMBH, approaches the SMBH until it reashes a distance comparable to the "tidal radius", $r_{t}:=a\left(M_{3} / m_{12}\right)^{1 / 3}$, where $a$ is the semimajor axis of the binary, $M_{3}$ is the mass of the SMBH and $m_{12}$ is the total mass of the binary. Moreover, the binary initially should have a small semimajor axis so that after being captured it remains tightly bound to and not easily breakable from the SMBH even though it repeatedly interacts with the background stars. This condition for stability imposes to $a$ an upper limit $a_{\text {cri }}$ which depends on the relaxation timescale $T_{\mathrm{rlx}}$ of the NSC.

Using the $a_{\text {cri }}$ derived in Chen \& Han (2018) and assuming that the initial pericentre of the COM of the BBH is $\xi r_{t}$ where $\xi$ is a factor of order unity, we can derive the minimum distance of the captured BBHs as

$$
\frac{r}{R_{S}} \simeq \frac{15(\xi q)^{3 / 8}}{(1+q)^{1 / 4}}\left(\frac{T_{\mathrm{rlx}}}{10^{9} \mathrm{yrs}}\right)^{1 / 4}\left(\frac{m_{1}}{10 M_{\odot}}\right)^{1 / 2}\left(\frac{M_{3}}{10^{6} M_{\odot}}\right)^{-3 / 4}
$$

where $m_{1}$ and $m_{2}$ are the masses of the two small BHs (we assume $m_{1} \geq m_{2}$ ), and $q:=m_{2} / m_{1}$ is their mass ratio. Only $1 \%$ of the captured binaries may coalesce at this distance according to preliminary results from numerical simulations, and hence the event rate is too low to explain the LIGO/Virgo detections unless the capture rate of BBHs has been significantly underestimated in the current models (Chen \& Han 2018).

The second mechanism is based on the scenario of $\mathrm{BBH}$ formation in AGN accretion discs (Syer et al. 1991; McKernan et al. 2018). After BBHs form in the disc, the hydrodynamical interaction with the surrounding gas drives them to

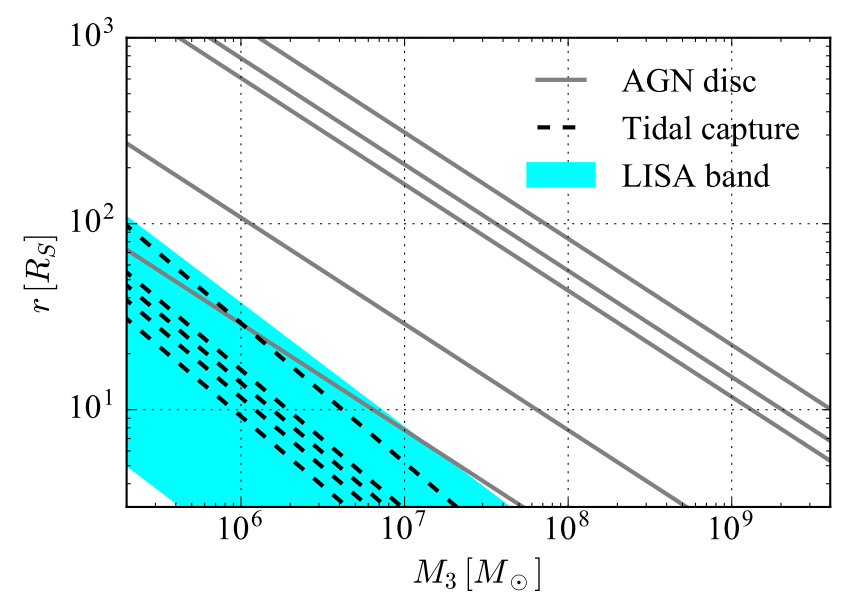

Figure 1. Predicted location for $\mathrm{BBH}$ mergers as a function of the mass of the central SMBH. The fiducial parameters are $m_{1}=$ $10 M_{\odot}, q=1, T_{\mathrm{rlx}}=10^{9}$ years, $\xi=2$ and $\dot{m}=1$. The dashed lines are derived from Equation (1), which corresponds to the tidal-capture model (Chen \& Han 2018). From top to bottom they refer to the results for, respectively, (1) $m_{1}=20 M_{\odot}$, (2) the fiducial model, (3) $\xi=1.3$, (4) $q=0.3$ and (5) $T_{\mathrm{rlx}}=10^{8}$ years. The solid lines are computed using Equation (2), i.e., the equilibrium radius for a BBH embedded in an AGN disc (Chakrabarti 1993). From top to bottom they correspond to (1) $m_{1}=20 M_{\odot}$, (2) the fiducial model, (3) $q=0.3$, (4) $\dot{m}=10^{3}$ and (5) $\dot{m}=10^{5}$. BBHs falling in the cyanshaded area emit GWs in the frequency band of $10^{-2}-10^{-4} \mathrm{~Hz}$ (see, e.g., Chen \& Amaro-Seoane 2017; Han \& Chen 2018) and hence are detectable by a LISA-type interferometer.

coalescence (Stone et al. 2017; Bartos et al. 2017). Several locations in the disc can trap stellar BHs for a long time (Chakrabarti 1993; Bellovary et al. 2016), and hence they are the preferential places for BBH mergers.

In particular, when the accretion rate is comparable or exceeding the Eddington limit, the inner part of the disc becomes super-Keplerian (Abramowicz \& Fragile 2013) so that a stellar $\mathrm{BH}$ embedded in it gains angular momentum as it accretes from the surrounding gas. This accretion compensates the angular-momentum loss via the GW radiation generated by the orbit of the small $\mathrm{BH}$ around the $\mathrm{SMBH}$ (Chakrabarti 1993). An equilibrium could be achieved at a radius of

$\frac{r}{R_{S}} \simeq 15 \dot{m}^{-2 / 7}\left(\frac{M_{3}}{10^{9} M_{\odot}}\right)^{-4 / 7}\left(\frac{m}{20 M_{\odot}}\right)^{4 / 7}$

(Chakrabarti 1993), where $m$ is the mass of the small body embedded in the disc and in our problem we set $m=m_{12}$. The accretion rate of the small body, $\dot{m}$, is normalized by the Eddington rate. We note that the event rate of $\mathrm{BBH}$ mergers in this scenario is unknown and deserves a future investigation.

Figure 1 illustrates the results from Equations (1) and (2). We can see that for the tidal-capture model (dashed lines) to produce BBHs at $r<10 R_{S}$, the SMBHs should be about $10^{6-7} M_{\odot}$. We notice that most of the SMBHs detected in the local universe fall in this mass range (Kormendy \& Ho 2013). Regarding the accretion-disc model (solid lines), if we assume $m_{1} \simeq m_{2} \simeq 10 M_{\odot}$, we find that $r \lesssim 10 R_{S}$ when $M_{3} \gtrsim 2.0 \times 10^{9} \dot{m}^{-1 / 2} M_{\odot}$. If we further consider the results 
from the recent studies which showed that $1 \lesssim \dot{m} \lesssim 10^{5}$ for the stellar BHs inside AGN accretion discs (Inayoshi et al. 2016; Stone et al. 2017), we can conclude that $M_{3}$ has to be at least $6.3 \times 10^{6} M_{\odot}$ to produce BBH merge at $r \stackrel{\sim}{<10} R_{S}$.

Figure 1 also shows that both the tidally captured BBHs and those most-rapidly accreting binaries in AGN discs are settled on such tightly bound orbits around SMBHs that their orbital motion will generate GWs detectable by the Laser Interferometer Space Antenne (LISA). This result implies that future joint observations of GWs using both LIGO/Virgo and LISA and help us identify BBH mergers happening very close to SMBHs (Han \& Chen 2018).

\section{EFFECTS OF REDSHIFT ON THE MEASUREMENT OF MASS}

If BBHs coalesce within $10 R_{S}$ of SMBHs, the Doppler and gravitational redshift could significantly affect the interpretation of the observational data. In GW astronomy, the observables are the amplitude $h$ (in two polarizations), frequency $f$, and the rate at which the signal chirps $\dot{f}$. If spacetime is flat without redshift effect, one can measure $f$ and $\dot{f}$ in the "inspiral" part of the waveform, where the BHs are far apart so that they can be approximated by point masses, and infer from them an intrinsic mass scale for the system,

$\mathcal{M}:=\left(\frac{5 f^{-11 / 3} \dot{f}}{96 \pi^{8 / 3}}\right)^{3 / 5}$.

This is known as the "chirp mass" because it determines how the frequency increases with time. Theoretically, it depends on the masses of the two BHs as $\mathcal{M}=\left(m_{1} m_{2}\right)^{3 / 5}\left(m_{1}+m_{2}\right)^{-1 / 5}$. Together with the third observable $h$, one can further infer the (luminosity) distance of the source (Holz \& Hughes 2005), using

$d=(4 \mathcal{M} / h)(\pi f \mathcal{M})^{2 / 3}$.

Redshift, by definition, reduces the frequency of a wave from a value of $f$, measured close to the source, to a value of $f_{o}=f(1+z)^{-1}$, where the subscript $o$ indicates a quantity measured in the rest frame of an observer. Correspondingly, the chirp rate changes from $\dot{f}$ to $\dot{f}_{o}=\dot{f}(1+z)^{-2}$, where the additional $(1+z)$ factor comes from time dilation. As a result, the only chirp mass that an observer can construct from the observed waveform is

$\mathcal{M}_{o}:=\left(\frac{5 f_{o}^{-11 / 3} \dot{f}_{o}}{96 \pi^{8 / 3}}\right)^{3 / 5}=\mathcal{M}(1+z)$.

It is greater than the intrinsic one by a redshift factor. Without an independent measurement of $z$, there is no way of disentangling $\mathcal{M}$ and $1+z$. This is a famous problem called "the mass-redshift degeneracy", and this effect applies to all three kinds of redshift, namely, the cosmological, Doppler and gravitational redshift.

\section{EFFECTS ON THE MEASURE OF DISTANCE}

Starting from the observables, the only possible way of constructing a distance scale is via

$d_{o}:=\left(4 \mathcal{M}_{o} / h_{o}\right)\left(\pi f_{o} \mathcal{M}_{o}\right)^{2 / 3}$, where $h_{o}$ is the observed amplitude. The distances of the LIGO/Virgo BBHs are derived effectively in this way. Therefore, it is crucial, for the purpose of this work, to understand what $d_{o}$ really is.

(1) Cosmological redshift: The expansion of the universe causes a redshift, $z_{\cos }$, which increases with the transverse comoving distance $d_{C}$. The GW amplitude damps linearly with $d_{C}$, i.e.

$h_{o}=\left(4 \mathcal{M} / d_{C}\right)(\pi f \mathcal{M})^{2 / 3}$.

Using the last equation to replace $h_{o}$ in Equation (6) and noticing that (a) $d_{C}\left(1+z_{\cos }\right)$ is the luminosity distance $d_{L}$ in a flat universe and (b) $f \mathcal{M}=f_{o} \mathcal{M}_{o}$, we find that $d_{o}=d_{C}\left(1+z_{\mathrm{cos}}\right)=d_{L}$. Therefore, we recover the classical notion that GWs encode the luminosity distance of the source (Schutz 1986).

(2) Doppler redshift: The relative motion of a GW source with respect to an observer also causes a shift to the observed frequency. It will be a redshift if the source is receding relative to the observer and a blueshift if the source is approaching. For simplicity we restrict ourselves to the case in which the COM of the $\mathrm{BBH}$ is moving at a constant velocity of $v$ away from the observer. According to the theory of special relativity, the Doppler redshift is

$1+z_{\mathrm{dop}}=\gamma(1+\beta)$

where $\beta=v / c$ and $\gamma=\left(1-\beta^{2}\right)^{-1 / 2}$ is the Lorentz factor.

Now the total redshift becomes $1+z_{\text {tot }}=\left(1+z_{\text {cos }}\right)\left(1+z_{\text {dop }}\right)$, and, correspondingly, we have $f_{o}=f\left(1+z_{\text {tot }}\right)^{-1}$ and $\dot{f}_{o}=$ $\dot{f}\left(1+z_{\text {tot }}\right)^{-2}$. The redshifted mass, as can be measured from GWs, consequently, becomes $\mathcal{M}_{o}=\mathcal{M}\left(1+z_{\cos }\right)\left(1+z_{\text {dop }}\right)$.

As for $h_{o}$, numerical simulations show that to linear order it is not affect by the Doppler effect (Gerosa \& Moore 2016). We note that this result is derived for plane waves and may not be applicable to spherical waves. Therefore, in the plane-wave approximation $h_{o}$ equals that $h$ in Equation (7). Using these new relations to replace $\mathcal{M}_{o}, f_{o}$, and $h_{o}$ in Equation (6), we find that $d_{o}=d_{C}\left(1+z_{\cos }\right)\left(1+z_{\mathrm{dop}}\right)=d_{L}\left(1+z_{\mathrm{dop}}\right)$. This result shows that the apparent distance $d_{o}$ is an overestimation of the real (luminosity) distance by a factor of $1+z_{\text {dop }}$

(3) Gravitational redshift: Waves originating from a deep gravitational potential also get redshifted. Since we are interested in GWs from the vicinity of a $\mathrm{SMBH}$, our gravitational potential reduces to that of a point mass. The spacetime surrounding it can be described by the Schwarzschild metric assuming the simple case that the hole is not rotating. In the following we restrict our discussions to $r>4 M_{3}$, because it is a limit imposed by the innermost bound orbit.

To derive the redshift for GWs, we first notice that $M_{3} / m_{12}>10^{5}$ for our problem, since $M_{3} \gtrsim 10^{6} M_{\odot}$ according to our earlier analysis and we are interested in BBHs with $m_{1} \sim m_{2} \sim 10 M_{\odot}$. This disproportion has two consequences that significantly simplify our problem. First, we are interested in the GWs emitted during the last few cycles of a merger because BBHs enter the LIGO/Virgo band when their semimajor axes become $a \lesssim 10 m_{12}$. Therefore, we are in a regime where the curvature radius of the background space-time, $\rho \sim \sqrt{r^{3} / M_{3}}>8 M_{3}$ (Isaacson 1968), is more than $10^{5}$ times greater than the wavelength of the gravitational radiation, which is comparable to $a$. Second, the waveform of the merger stretches over a time interval of $\Delta t \sim 2 \pi \sqrt{a^{3} / m_{12}}$, 
which corresponds to a length of $\Delta l=c \Delta t$. This length is only a fraction of $\Delta l /\left(2 M_{3}\right) \lesssim \pi \sqrt{10^{3}}\left(m_{12} / M_{3}\right) \lesssim 2 \times 10^{-3}$ of the length scale of the Schwarzschild metric $\left(2 M_{3}\right)$.

From the above arguments we conclude that we are in a short-wave limit. In this case, GWs propagate in approximately the same way as light waves (Isaacson 1968). So we can use the redshift for photons,

$1+z_{\text {gra }}=\left(1-R_{S} / r\right)^{-1 / 2}$,

to calculate the GW frequencies in the rest frame of the observer, who is supposedly at infinity ("final observer" hereafter). The last equation is appropriate only for the case in which the source and observer are on the same side of the SMBH. Otherwise, the GWs have to circle around the SMBH to get to the observer, in which case the waves could have reached a closer distance to the SMBH. Strong lensing effect is expected in the latter case (Kocsis 2013), but our analytical scheme cannot treat it properly. Therefore, we take the simple case for illustrative purposes and find

$1+z_{\mathrm{tot}}=\left(1+z_{\mathrm{cos}}\right)\left(1+z_{\mathrm{dop}}\right)\left(1+z_{\mathrm{gra}}\right)$

and

$\mathcal{M}_{o}=\mathcal{M}\left(1+z_{\mathrm{cos}}\right)\left(1+z_{\mathrm{dop}}\right)\left(1+z_{\mathrm{gra}}\right)$.

As for the GW amplitude, we start our analysis from the point of view of an intermediate observer at a small radial offset of $\Delta r \sim 10^{2} m_{12}$ from the COM of the binary. On one hand, this offset is much larger than the size of the binary and hence the wave front is not any more subject to distortion by the binary-the wave is fully developed (Kocsis \& Loeb 2007). On the other, $\Delta r /\left(2 M_{3}\right) \lesssim 50\left(m_{12} / M_{3}\right) \lesssim 10^{-3}$, which means that it is a small offset relative to the length scale $\left(2 M_{3}\right)$ of the coordinates. As a result, from $r$ to $r+\Delta r$ we do not need to consider the effect of redshift on the GW amplitude. Furthermore, the corresponding proper distance, $\Delta d \simeq \Delta r / \sqrt{1-2 M_{3} / r}<\sqrt{2} \Delta r$, is only a small fraction of $\lesssim 4 \times 10^{-4}$ of the curvature radius $\rho$, and hence the intermediate observer is conducting an observation in an effectively flat space-time. The small redshift and the flatness of space-time allow us to use the conventional formula $h \simeq 4(\mathcal{M} / \Delta d)(\pi f \mathcal{M})^{2 / 3}$ to derive the $\mathrm{GW}$ amplitude at the location of the intermediate observer.

As the wave passes the intermediate observer and propagates towards the final observer, the amplitude decreases linearly with the proper distance, $d_{p}$. In the end the final observer detects an amplitude of $h_{o} \simeq h\left(\Delta d / d_{p}\right)=$ $4\left(\mathcal{M} / d_{p}\right)(\pi f \mathcal{M})^{2 / 3}$, where we have assumed $\Delta d \ll d_{p}$. In principle the calculation of $d_{p}$ can be separated into two parts. The first part deals with the proper distance close to the SMBH which is different from the coordinate distance because of the Schwarzschild metric. The second part is the cosmological comoving distance from the SMBH to the final observer $d_{C}$. Since the BBH normally is far from the event horizon of the SMBH, the cosmological distance is the dominant component. Therefore, we can neglect the first part of $d_{p}$ and write $d_{p} \simeq d_{C}$. Finally, we find that $h_{o}$ reduces to that in Equation (7).

To complete our analysis, we use the $h_{o}, \mathcal{M}_{o}$, and $f_{o}=$ $f /\left(1+z_{\text {tot }}\right)$ derived in this section to rewrite Equation (6).
We find that

$$
\begin{aligned}
d_{o} & =d_{C}\left(1+z_{\mathrm{cos}}\right)\left(1+z_{\mathrm{dop}}\right)\left(1+z_{\mathrm{gra}}\right) \\
& =d_{L}\left(1+z_{\mathrm{dop}}\right)\left(1+z_{\mathrm{gra}}\right) .
\end{aligned}
$$

Therefore, the apparent distance $d_{o}$ is even bigger when the gravitational redshift is added into the analysis.

\section{THE MAXIMUM EFFECT}

To estimate the upper limit of $z_{\text {tot }}$, we need to find the smallest $r$. For a circular orbit, which would be relevant for the BBHs trapped in AGN accretion discs, the smallest $r$ is imposed by the innermost stable circular orbit (ISCO), i.e. $r=6 M_{3}$ for a non-rotating SMBH. In this case, the gravitational redshift is $1+z_{\text {gra }} \simeq 1.22$ according to Equation (9). The circular velocity according to the Keplerian approximation is $v \simeq c / \sqrt{6} \simeq 0.408 c$, so the Doppler redshift is $1+z_{\text {dop }} \simeq 1.54$ according to Equation (8). The total redshift due to these two effects is $\left(1+z_{\text {dop }}\right)\left(1+z_{\text {gra }}\right) \simeq 1.89$,

On the other hand, if the orbit around the $\mathrm{SMBH}$ is nearly parabolic, a more likely configuration in the model of tidal capture, it is the innermost bound orbit (IBO), i.e. $r=4 M_{3}$, that is limiting $r$. In this case, the gravitational redshift increases to $1+z_{\text {gra }} \simeq 1.41$ and a maximum velocity of $c / \sqrt{2} \simeq 0.707 c$ can be reached at the pericentre passage, which corresponds to a Doppler redshift of $1+z_{\text {dop }} \simeq 2.41$. Consequently, the total redshift increases to $1+z_{\text {tot }} \simeq 3.41$.

Therefore, the effect caused by Doppler and gravitational redshifts is at most $1+z_{\text {tot }} \simeq(1.9-3.4)$. Interestingly, these values coincide with the contrast between the typical mass of the BHs detected by LIGO/Virgo $\left(20-30 M_{\odot}\right)$ and the canonical mass of the BHs in X-ray binaries $\left(10 M_{\odot}\right)$. This coincidence can be more clearly seen in Figure 2, where we plot the observed chirp masses of the ten detected BBHs against their apparent distances. We color code the data points according to the masses of the $\mathrm{BHs}$ prior to the merger. If one $\mathrm{BH}$ member prior to the merger is greater than $20 M_{\odot}$, we plot the binary as a red dot. Otherwise, if both $\mathrm{BHs}$ are lighter than $20 M_{\odot}$, we plot the binary as a blue dot. We choose $20 M_{\odot}$ as the threshold because so far there is no stringent detection in X-ray binaries of BHs more massive than this mass (McClintock et al. 2014; Corral-Santana et al. 2016).

The $d_{o}-\mathcal{M}_{o}$ diagram reveals two striking features. (1) There is a gap between the red and blue populations. It is even more evident when we plot in Figure 3 the mass of each $\mathrm{BH}$ prior to the merger. In the light of our redshift model, a gap is expected because the red population are highly redshifted, so that they should have been displaced in the diagonal direction, from the location occupied by the blue population to a region of higher mass and larger distance. (2) If we use a hypothetical redshift of $1+z_{\text {tot }}=3.4$ to estimate the "intrinsic" chirp masses and luminosity distances for the BBHs in the red population, we find the grey dots in Figures 2 and 3. This grey population occupy the same region in the diagram as the blue one. This result is, again, consistent with the hypothesis that the red population have been redshifted. We note that the robustness of the features shown here is limited by the current small-number statistics, but future detection of more BBHs can help us further test our hypothesis. 


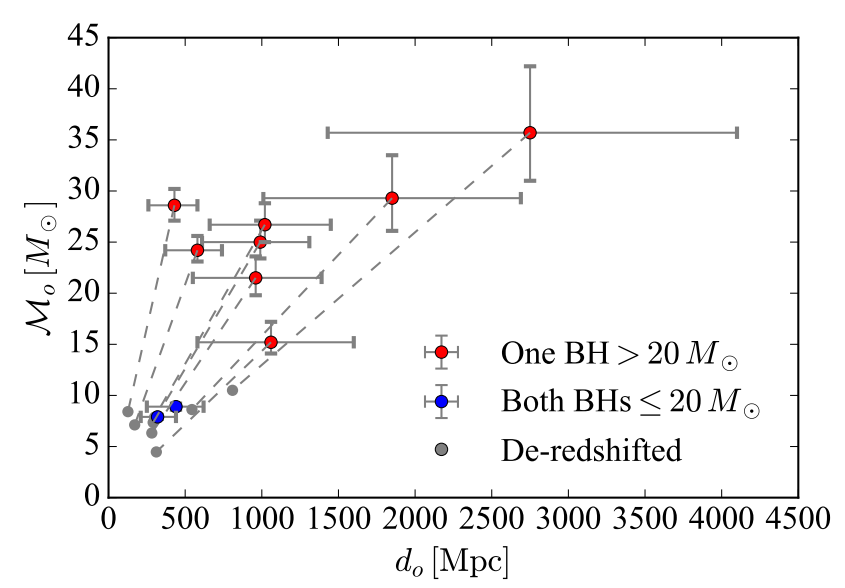

Figure 2. The observed chirp masses versus the apparent distances for the ten BBHs detected by LIGO/Virgo (The LIGO Scientific Collaboration \& The Virgo Collaboration 2018a). The red dots refer to the binaries which contain at least one $\mathrm{BH}$ member with a mass greater than $20 M_{\odot}$. The blue ones refer to those binaries whose $\mathrm{BH}$ members are all lighter than $20 M_{\odot}$. The grey dots attached by dashed lines show the hypothetical chirp masses and distances derived using the maximum redshift factor of $1+z=3.4$.

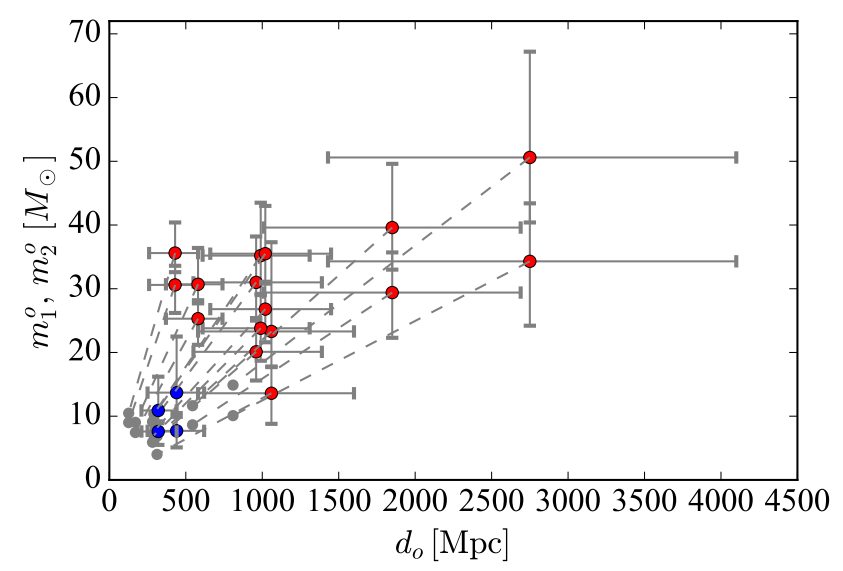

Figure 3. The same as Figure 2 but showing the mass of each $\mathrm{BH}$ prior to the merger.

\section{DISCUSSION}

In this Letter we have shown that the current astrophysical models allow BBHs to form and merge at a distance of $r \lesssim$ $10 R_{S}$ from a SMBH (Section 2). In this case, the emitted GWs would be affected by the Doppler and gravitational redshift in such a away that the mass and distance derived from the observed waveform are greater than their intrinsic values by a redshift factor of $1+z_{\text {tot }}$ (Sections 3 and 4 ). In the most extreme scenario considered for non-spinning Schwarzschild SMBHs, $1+z_{\text {tot }}$ could be as large as $1.9-3.4$ and, interestingly, this value agrees with the data of the ten BBHs detected so far by LIGO/Virgo (Section 5).

In the case of non-spinning SMBHs, because the upper limit of $\left(1+z_{\text {dop }}\right)\left(1+z_{\text {gra }}\right)$ is 3.4 and the maximum BH mass de- tected in X-ray binaries so far is about $20 M_{\odot}$, we expect that the $\mathrm{BHs}$ detected by LIGO/Virgo at relative small distance (so that we know $z_{\text {cos }}$ is small) would not be more massive than $70 M_{\odot}$ before their mergers. If most $\mathrm{SMBHs}$ are highly spinning, the maximum value of $\left(1+z_{\text {dop }}\right)\left(1+z_{\text {gra }}\right)$ could be even higher, although the exact value deserves a more robust calculation. These predictions can be tested against future GW observations.

Apart from the redshift effect, other types of distortion of the inspiraling waveform caused by the presence of a $\mathrm{SMBH}$ is relatively weak. (i) The tidal force of the SMBH is unimportant when the BBH enters the LIGO/Virgo band. This is so because the duration of the merger event in the band, i.e. $\Delta t$ derived in the previous section, is much shorter than the Lidov-Kozai timescale $t_{\mathrm{KL}}$ (see Naoz et al. 2013, for details). In fact, in our problem where $r \sim 6 M_{3}, a \lesssim 10 m_{12}$ and $M_{3} \gtrsim 10^{6} M_{\odot}, t_{\mathrm{KL}}$ is at least $10^{-2}\left(M_{3} / m_{12}\right)^{2} \simeq 3 \times 10^{7}$ times longer than $\Delta t$. (ii) The motion of the BBHs around the SMBHs is also insignificant during the merger. In principle, the motion induces a time-dependent gravitational background which leads to a further phase drift of the waveform (Meiron et al. 2017; Inayoshi et al. 2017). But in our case, the merger time $\Delta t$ is very short, such that during this interval the COM of the binary shifts by only a length of $v \Delta t \lesssim 230 m_{12}$, which is more than 400 times smaller than the length scale $2 M_{3}$ of the local metric. Therefore, the phase drift is negligible in our problem. We note that phase drift becomes important only when $M_{3} \lesssim 10^{4} M_{\odot}$, because the ratio $v \Delta t /\left(2 M_{3}\right)$ becomes greater than 0.2. But for our problem of $M_{3} \gtrsim 10^{6} M_{\odot}$, the background is essentially adiabatic.

The most critical problem of the tidal-capture model is that the event rate seems too low to explain all the eight massive stellar BHs detected by LIGO/Virgo. For example, the merger rate is estimated to be at most $0.03 \mathrm{Gpc}^{-3} \mathrm{yr}^{-1}$ (Chen \& Han 2018), while the rate inferred from the LIGO/Virgo detections is $\left(10-10^{2}\right) \mathrm{Gpc}^{-3} \mathrm{yr}^{-1}$ (The LIGO Scientific Collaboration \& The Virgo Collaboration 2018a). One possible mitigation of the tension is that the capture rate of BBHs by SMBH could have been significantly underestimated. It is possible because the previous estimation is based on the (loss-cone) theory which regards BBHs as point masses. In this way, the diffusion of the binaries in the phase space of energy and angular momentum around the SMBHs can be studied using the conventional models developed for single stars. In reality, however, binaries exchange energy and angular momentum with background stars in a way more efficiently than the conventional two-body interactions (Heggie 1975). For this reason, the capture rate deserves a re-evaluation.

The event rate of the BBH mergers in AGN accretion discs is more uncertain. Possible values range from $O(1) \mathrm{Gpc}^{-3} \mathrm{yr}^{-1}$ (Bartos et al. 2017; Stone et al. 2017) to as large as $10^{4} \mathrm{Gpc}^{-3} \mathrm{yr}^{-1}$ (McKernan et al. 2018). However, the models which lead to these values have not included the possibility that BBHs could be trapped at a distance as small as $r \lesssim 10 R_{S}$ from a SMBH (Chakrabarti 1993, and also see our Eq. 2). We plan to look into this possibility and calculate the corresponding merger rate in a future work. 


\section{ACKNOWLEDGEMENTS}

We thank Fukun Liu, Runqiu Liu, Kejia Lee, Rainer Spurzem, Zoltan Haiman, Bence Kosis and Alberto Sesana for useful discussions. This work is supported by the "985 Project" of Peking University and the NSFC grants No. 11873022, 11773059, 11303039, 11622546 and 11375260. $\mathrm{XC}$ and SL are partly supported by the Strategic Priority Research Program of the Chinese Academy of Sciences through the grants No. XDB23040100 and XDB23010200, and by the Silk Road Project of the National Astronomical Observatories, Chinese Academy of Sciences.

\section{REFERENCES}

Abbott B. P., et al., 2016, ApJ, 818, L22

Abramowicz M. A., Fragile P. C., 2013, LRL, 16, 1

Addison E., Laguna P., Larson S., 2015, preprint, (arXiv:1501.07856)

Alexander T., Hopman C., 2009, ApJ, 697, 1861

Amaro-Seoane P., Chen X., 2016, MNRAS, 458, 3075

Amaro-Seoane P., Freitag M., Spurzem R., 2004, MNRAS, 352, 655

Antonini F., Perets H. B., 2012, ApJ, 757, 27

Arca-Sedda M., Gualandris A., 2018, MNRAS, 477, 4423

Bahcall J. N., Wolf R. A., 1976, ApJ, 209, 214

Bartos I., Kocsis B., Haiman Z., Márka S., 2017, ApJ, 835, 165

Bellovary J. M., Mac Low M.-M., McKernan B., Ford K. E. S., 2016, ApJ, 819, L17

Bradnick B., Mandel I., Levin Y., 2017, MNRAS, 469, 2042

Broadhurst T., Diego J. M., Smoot George I., 2018, arXiv eprints, p. arXiv:1802.05273

Chakrabarti S. K., 1993, ApJ, 411, 610

Chen X., Amaro-Seoane P., 2017, ApJ, 842, L2

Chen X., Han W.-B., 2018, Comms. Phys., 1, 53

Corral-Santana J. M., Casares J., Muñoz-Darias T., Bauer F. E., Martínez-Pais I. G., Russell D. M., 2016, A\&A, 587, A61

Freitag M., Amaro-Seoane P., Kalogera V., 2006, ApJ, 649, 91

Gerosa D., Moore C. J., 2016, PRL, 117, 011101

Han W.-B., Chen X., 2018, arXiv e-prints:1801.07060,

Heggie D. C., 1975, MNRAS, 173, 729

Hoang B.-M., Naoz S., Kocsis B., Rasio F. A., Dosopoulou F., 2018, ApJ, 856, 140

Holz D. E., Hughes S. A., 2005, ApJ, 629, 15

Hopman C., Alexander T., 2006, ApJ, 645, L133

Inayoshi K., Haiman Z., Ostriker J. P., 2016, MNRAS, 459, 3738

Inayoshi K., Tamanini N., Caprini C., Haiman Z., 2017, PRD, 96, 063014

Isaacson R. A., 1968, Phys. Rev., 166, 1263

Kocsis B., 2013, ApJ, 763, 122

Kocsis B., Loeb A., 2007, PRD, 76, 084022

Kormendy J., Ho L. C., 2013, ARA\&A, 51, 511

Liu B., Wang Y.-H., Yuan Y.-F., 2017, MNRAS, 466, 3376

McClintock J. E., Narayan R., Steiner J. F., 2014, SSRv, 183, 295

McKernan B., et al., 2018, ApJ, 866, 66

Meiron Y., Kocsis B., Loeb A., 2017, ApJ, 834, 200

Miller M. C., Lauburg V. M., 2009, ApJ, 692, 917

Miralda-Escudé J., Gould A., 2000, ApJ, 545, 847

Morris M., 1993, ApJ, 408, 496

Naoz S., Kocsis B., Loeb A., Yunes N., 2013, ApJ, 773, 187

Petrovich C., Antonini F., 2017, ApJ, 846, 146

Prodan S., Antonini F., Perets H. B., 2015, ApJ, 799, 118

Schutz B. F., 1986, Nat, 323, 310

Smith G. P., Jauzac M., Veitch J., Farr W. M., Massey R., Richard J., 2018, MNRAS, 475, 3823
Stephan A. P., Naoz S., Ghez A. M., Witzel G., Sitarski B. N., Do T., Kocsis B., 2016, MNRAS, 460, 3494

Stone N. C., Metzger B. D., Haiman Z., 2017, MNRAS, 464, 946 Syer D., Clarke C. J., Rees M. J., 1991, MNRAS, 250, 505

The LIGO Scientific Collaboration the Virgo Collaboration 2018, arXiv e-prints, p. arXiv:1811.12907

The LIGO Scientific Collaboration The Virgo Collaboration 2018, arXiv e-prints, p. arXiv:1811.12940

VanLandingham J. H., Miller M. C., Hamilton D. P., Richardson D. C., 2016, ApJ, 828, 77

This paper has been typeset from a $\mathrm{T}_{\mathrm{E}} \mathrm{X} / \mathrm{LAT} \mathrm{T} \mathrm{X}$ file prepared by the author. 